\title{
Informationstagung Mikroelektronik 2003
}

Die Informationstagung Mikroelektronik 2003, welche seit dem Jahre 1975 im Zweijahresrhythmus im Rahmen der "Internationalen Fachmesse für Elektrotechnik, Elektronik, Licht, Automation und Antriebstechnik (viet)" stattindet, ist die 15. Veranstaltung in ihrer Reihe.

Die Informations- und Kommunikationstechnik wächst unbeirrt und beeinflusst unsere Gesellschaft in ungeahnter Weise. Die Basis hiefür bildet die Mikroelektronik, die durch ihre rasante Entwicklung, was Leistung, Energiebedarf und Kosten der ICs betrifft, aber auch durch entsprechende hervorragende Applikationen in der Kommunikation, in der Mess-, Steuerungsund Regelungstechnik sowie in der Schaltungstechnik (Optoelektronik usw.) dieses ermöglicht. Zur Absicherung des zukünftigen Wachstums unternimmt die Halbleiterindustrie gewaltige Anstrengungen zur Weiterentwicklung ihrer Produktionstechnologien, die den immer höheren Leistungsansprüchen und der dadurch bedingten Schaltungsdichte gerecht werden müssen.

Die Informationstagung Mikroelektronik kann aus der Fülle dieser Entwicklungen jeweils nur einen kleinen Ausschnitt bieten, welcher aber in eindrucksvoller Weise die dramatische Steigerung der Leistungsfähigkeit dieser Technologie darstellt. Es ist auch diesmal wieder gelungen, eine Vielzahl von hoch interessanten Vorträgen und Posterbeiträgen zu erhalten, wodurch diese Veranstaltung sicherlich einen ausgezeichneten Beitrag zum Stand der Technik in den ausgewählten Themenkreisen leisten wird: Die in diesem Heft der e \& i veröffentlichten Hauptvorträge dokumentieren dies in eindrucksvoller Weise. Die ebenfalls qualitativ hochwertigen Kurzvorträge und Posterbeiträge werden im Tagungsband der ME 03 veröffentlicht, welcher, wie in den letzten Jahren, als Band der Schriftenreihe des OVE herausgegeben wird.

Um inhaltlich eine größere Komplexität und Geschlossenheit zu gewährleisten, hat das wissenschaftliche Redaktionskomitee für die diesjährige Tagung drei Themenkreise ausgewählt, welche für die heutige Zeit von großer Bedeutung sind und in fünf Hauptvorträgen, 15 Kurzvorträgen und 74 Posterpräsentationen samt Beiträgen für den Tagungsband abgehandelt werden.

Neben dem Eröffnungsvortrag von Prof. Dr. Claus Weyrich (Siemens-München) mit dem Titel: Die Zukunft der Informations- und Kommunikationstechnik - Visionen, Trends, Technologien, wurden folgende drei Themenkreise gewählt:

(1) Nanotechnologie

(2) Embedded Systems

(3) Wireless Access

Darüber hinaus findet das bereits anlässlich der letzten Tagungen eingeführte "Informationsforum Mikroelektronik" statt. Dieses Informationsforum wird wieder von O. Univ.-Prof. Dipl.Ing. Dr. Gottfried Magerl geleitet und rundet obige Themenkreise mit einem Round Table-Gespräch zum Thema „The intelligent highway" ab. Für dieses Gespräch konnten als Teilnehmer namhafte Repräsentanten der Industrie gewonnen werden.

Die Informationstagung Mikroelektronik 2003 wird weiters ergänzt durch ein Workshop mit dem Titel „Softwareentwicklung für Dependable Embedded Systems“. Diese Parallelveranstaltung liefert Beiträge und Diskussionen zum Thema Herausforderungen, "Roadmap“ und Vision einer Technologie, Zusammenhang mit dem laufenden Call des 6 . Rahmenprogramms. Die Beiträge dieser Veranstaltung werden ebenfalls im Tagungsband veröffentlicht.

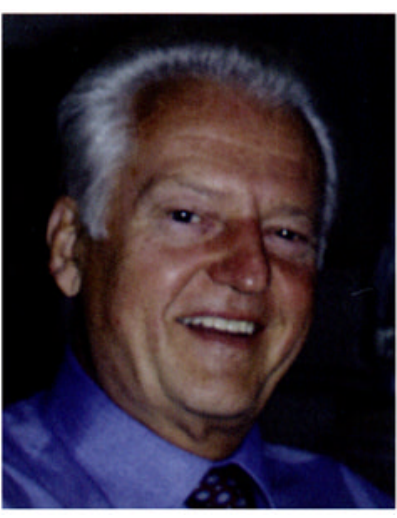

Hofrat Dipl.-Ing. Günther Fiedler

Diese Tagung war und ist immer darauf ausgerichtet, Entwickler, Wissenschaftler und Konsumenten zusammen zu bringen. Die Mikroelektroniktagung, welche immer im Rahmen dieser Messeveranstaltung stattgefunden hat und stattindet, hat nie den Anspruch erhoben, wissenschaftliche Ergebnisse auf höchstem Niveau zu veröffentlichen. Alleine der Name Informationstagung besagt schon, dass Interessierte informiert werden sollen. Diese Tagung widmet sich daher aktuellen Fragen und Problemlösungen, welche sich durch die Entwicklung und Anwendung der Mikroelektronik ergeben. Anschließend ist jeweils eine eingehende Diskussion zwischen den Autoren und den Tagungsteilnehmern vorgesehen.

Ziel dieser Veranstaltung ist es also, in den genannten Themenkreisen einen Überblick über den Stand der Technik zu vermitteln. Ein Erfahrungsaustausch zwischen Wissenschaftlern, Mitarbeiter in- und ausländischer Unternehmen, speziell der Entwicklungsingenieure, bzw. öffentlicher und schulischer Institutionen soll hergestellt werden. Eine Annäherung von Wissenschaft und Industrie (anwendungsorientierte Praxis) soll geschaffen werden, und Impulse für die Aus- und Weiterbildung sollen entstehen. Deshalb wird auch diesmal die Teilnahme an der Tagung kostenlos sein.

Durch die Integration des Tagungsortes in das Messegeschehen, nämlich im Messe Congress Center Wien, wird der Stellenwert dieser Tagung in Richtung Industrie noch stärker dokumentiert. Den Ausstellungsbesuchern wird dadurch auch vermehrt die Möglichkeit eingeräumt, an gezielten Themenkreisen der Tagung teilzunehmen.

Auch diesmal haben namhafte Vertreter der Industrie im wissenschaftlichen Redaktionskomitee mitgewirkt. Es ist damit wiederum die Einbeziehung der Industrie in das Tagungsgeschehen gewährleistet. Gerade die Integration der Tagung in die "viet" dokumentiert auch die Ausrichtung der Tagung in Richtung Industrie, ist doch die gegenseitige Information zwischen Forschung und Anwendung in dieser Zeit der rasanten Entwicklung besonders wichtig. Es ist zu hoffen und zu wünschen, dass die seitens der Forschung präsentierten Entwicklungen von der Industrie aufgegriffen werden und so neue Kooperationen zwischen Forschung und Wirtschaft entstehen.

Allerdings wäre das Zustandekommen der MikroelektronikTagung auch diesmal nicht ohne die tatkräftige Hilfe und Unterstützung seitens des Bundesministeriums für Verkehr, Innovation und Technologie sowie des Bundesministeriums für Bildung, Wissenschaft und Kultur möglich gewesen. 
An dieser Stelle muss auch dem Fachverband der Elektround Elektronikindustrie FEEI, dem Österreichischer Verband für Elektrotechnik OVE, den Firmen Austria Micro Systeme Int. AG, Infineon Technologies Int. AG, Mobilkom Austria AG und Telekom Austria AG, der Erste Bank der Österreichischen Sparkassen sowie im Besonderen der Reed Messe Wien gedankt werden. Zu Dank verpflichtet sind die Veranstalter schließlich auch der Schriftleitung der e \& i und dem Springer-Verlag für die ausgezeichnete Zusammenarbeit.

Ein besonderer Dank gebührt aber allen Autoren und Vortragenden, besonders denen der Hauptvorträge, sowie den Mitgliedern des wissenschaftlichen Redaktionskomitees der ME 03, welche sich alle sehr engagiert und unermüdlich eingesetzt haben. Insbesondere gilt auch mein Dank den Themenkreislei- tern, die die eingeladenen Vorträge organisierten und ohne deren Mitwirkung die Abwicklung einer solchen Tagung nicht möglich wäre.

Erfreulich ist auch die hohe Beteiligung aus dem benachbarten Ausland, das sowohl durch Vortragende als auch durch Posterpräsentationen vertreten ist.

Die Organisatoren hoffen, dass der Erfolg der Tagung die erbrachten Mühen der Vorbereitung rechtfertigt und wünschen den Teilnehmern eine für sie informative und nützliche Veranstaltung.

Hofrat Dipl.-Ing. Günther Fiedler

Tagungssekretär der Informationstagung Mikroelektronik 2003 Institut für Elektrische Mess- und Schaltungstechnik, Technische Universität Wien 